\title{
Case Report: Pazopanib Treatment Response in a Patient with Metastatic Pleomorphic Dermal Sarcoma (Atypical Fibroxanthoma) with Circulating Tumor Cell-Derived Colonies as a Predictive Marker
}

\author{
Wolfram E. Samlowski1 ${ }^{*}$, Joseph Wojcik ${ }^{1}$, Suzanne Samlowski², Douglas Fife ${ }^{3}$, Todd Murry ${ }^{4}$ \\ ${ }^{1}$ Comprehensive Cancer Centers of Nevada, Las Vegas, USA \\ ${ }^{2}$ TrueCells, LLC, Las Vegas, USA \\ ${ }^{3}$ Surgical Dermatology and Laser Center, Las Vegas, USA \\ ${ }^{4}$ LMC Pathology, Las Vegas, USA \\ Email: *wsamlowski1@gmail.com
}

How to cite this paper: Samlowski, W.E., Wojcik, J., Samlowski, S., Fife, D. and Murry, T. (2016) Case Report: Pazopanib Treatment Response in a Patient with Metastatic Pleomorphic Dermal Sarcoma (Atypical Fibroxanthoma) with Circulating Tumor CellDerived Colonies as a Predictive Marker. Journal of Cancer Therapy, 7, 785-793. http://dx.doi.org/10.4236/jct.2016.711079

Received: August 2, 2016

Accepted: October 28, 2016

Published: October 31, 2016

Copyright $\odot 2016$ by authors and Scientific Research Publishing Inc. This work is licensed under the Creative Commons Attribution International License (CC BY 4.0).

http://creativecommons.org/licenses/by/4.0/ (c) (i) Open Access

\begin{abstract}
Atypical fibroxanthomas (AFX) are rare skin tumors. These generally are superficial tumors, usually $<3 \mathrm{~cm}$ red, fleshy, ulcerated skin lesions, that characteristically occur on sun-damaged skin, sometimes in immunocompromised or previously irradiated patients. These are part of a spectrum of more aggressive fibro-histiocytic neoplasms. In the older literature, these have been termed aggressive or metastatic AFX, but currently these have been reclassified as pleomorphic dermal sarcomas (PDS) and systemic undifferentiated pleomorphic sarcoma (UPS, formerly malignant fibrohistiocytic sarcoma, MFH). We present the case of a 64-year old woman who developed a deeply invasive PDS on the vertex of her scalp invading to the galea, with in-transit scalp metastases. Very little information is available about optimal treatment of metastatic PDS lesions. The patient was initially treated with 2 cycles of epirubicin/ifosfamide chemotherapy, resulting in life-threatening complications. A pretreatment peripheral blood sample was sent for CTC-derived colony assay. This sample grew 8 colonies from 10 $\mathrm{ml}$ blood. The tumor failed to respond to epirubicin and ifosfamide, and after several months of hospitalization, a second peripheral blood CTC-derived colony assay grew $>376$ colonies. The patient could not tolerate additional chemotherapy. She was therefore treated with the oral targeted agent pazopanib. The patient developed a dramatic biopsy-confirmed complete response. After 11 months of pazopanib treatment, a repeat CTC-derived culture sample grew only 8 colonies $/ 10 \mathrm{ml}$ blood. The complete response to pazopanib is still ongoing at over 41 months. To our knowledge,
\end{abstract}


this is the first demonstration of clinical complete response of a PDS tumor following targeted therapy. An additional novel feature was the demonstration that CTC-derived colonies could be grown from the blood of a PDS patient. The number of colonies appeared to correlate with the clinical treatment response and seemed to function as a potential prognostic marker.

\section{Keywords}

Atypical Fibroxanthoma, Pleomorphic Dermal Sarcoma, Vascular Endothelial Growth Factor Receptor, Targeted Therapy, Circulating Tumor Cells, Circulating Tumor Cell-Derived Cultures

\section{Introduction}

Atypical fibroxanthomas (AFX) are uncommon spindle cell neoplasms that most often occur on the sun-exposed skin of older patients [1]. These tumors are generally less than $3 \mathrm{~cm}$ in size, and usually exhibit rapid initial growth [1]. Although patients with this tumor have a mean age of 69 years old, AFX has been described in patients ranging between ages 13 and 95. Recent studies report an increased incidence of AFX in younger immunosuppressed patients with AIDS, and organ transplantation. The anatomic distribution of deeply invasive lesions has been described as $58 \%$ extremities, $22 \%$ head and neck, $11 \%$ trunk, and 9\% retroperitoneal, mediastinal, or pelvis [2].

AFX tumor cells have a mesenchymal immunophenotype and consist of fusiform, epithelioid, and pleomorphic cells with numerous mitotic figures [3]. Multinucleate giant cells, osteoclastic giant cells, and foam cells are frequently present in atypical, more aggressive lesions. There is a characteristic accumulation of intracytoplasmic lipids [4].

AFX is histologically similar in appearance to other fibro-histiocytic malignancies that may involve the skin, such as pleomorphic dermal sarcoma (PDS) and undifferentiated pleomorphic sarcoma (UPS, formerly malignant fibrous histiocytoma or MFH). PDS currently is used to describe fibro-histiocytic skin malignancies that are limited to skin, but are larger, more deeply invasive tumors than the usual AFX, possibly with in-transit metastases. Deep subcutaneous invasion, tumor necrosis perineural or lymphovascular invasion may identify these more aggressive PDS tumors [5]. In contrast, distant organ metastases from a skin histio-fibroblastic primary are more consistent with a UPS diagnosis, although 25 cases of "metastatic AFX" are identified in the older literature [6]. In these rare patients, the reported sites of metastases have included lymph nodes (58\%), visceral organs (35\%), parotid glands (19\%), and local cutaneous sites (12\%), with some patients having metastases to multiple sites [7]. While PDS, like AFX usually develops on sun-exposed parts of the body, MFH most commonly occurs in the extremities.

It is virtually impossible to predict clinical behavior of AFX versus related tumors like PDS, and UPS, based on histologic appearance of the primary lesion. The size of the primary $(>3 \mathrm{~cm})$ and depth of tumor invasion appear to have prognostic value [5]. 
Tumor invasion into subcutaneous adipose tissues, muscle, or deeper tissues increases the risk for local recurrence and distant metastases [7]. Perineural and perivascular extension also appear to have adverse prognostic impact [5]. At a cellular level, there appear to be some differences. Most UPS tumors appear to have aneuploid DNA content, while AFX usually is diploid [7]. Immunohistology is used as a common method for attempting to differentiate spindle cell neoplasms. AFX generally exhibits increased expression of CD10 and excludes CD63, S100, and cytokeratin staining [1]. They also generally do not express CD34, distinguishing them from another spindle cell neoplasm, dermatofibrosarcoma protuberans (DFSP). Proposed molecular differences may also include increased Ki-67 staining in UPS tumors, as well as presence of H-RAS and K-RAS mutations in some MFH patients, in contrast to UV-related p53 mutations in some AFX tumors [7]. With the application of modern technology, it is hoped that there will be further improvement in classification [8].

Due to the similarities among AFX, PDS, and UPS, some consider AFX and PDS tumors to be a superficial variant of UPS. AFX tumors generally arise at sites of chronic ultraviolet radiation exposure to the skin, while the origins of UPS are poorly understood. UPS is considered to be the more malignant of the two conditions in that it may invade deeper tissues, and seems to have a greater metastatic potential, while AFX and PDS are considered more benign in their clinical behavior. This distinction may underestimate the potential aggressiveness of PDS tumors, which can certainly infiltrate the muscle and fascia and produce at least in-transit metastases.

We present a case of metastatic PDS in a 64-year old woman. Circulating tumor cell (CTC)-derived colonies were cultured from these samples using a novel technology. We demonstrated that CTC-derived cultures represented a potential marker for patient's response to treatment. In addition, this is the first report of a biopsy-confirmed complete response of a metastatic DPS to pazopanib therapy.

\section{Materials and Methods}

Thin sections of formalin fixed paraffin embedded tumor and skin biopsies were prepared by for hematoxylin and eosin staining and immunostaining with rabbit-anti-human monoclonal antibodies directed against CD10, SMA, S-100, pancytokeratin, CD34 and desmin using standard techniques.

\section{Growth of CTC-Derived Colonies}

The white cell fraction of each sample was separated by Ficoll/Hypaque centrifugation at $1500 \mathrm{G}$ for 15 minutes, then split into 8 replicate cultures in sterile $0.8 \%$ methylcellulose in RPMI 1640 medium with 1\% human serum, supplemented with non-essential amino acids [9]. After 16 days in culture, tumor colonies were counted.

\section{Case Report}

The patient is a 64-year old Caucasian woman with a long-standing history of rheumatoid arthritis, pyoderma gangrenosum, multiple prior squamous cell carcinomas, basal 
cell carcinomas, and one prior melanoma. Around January of 2012 she developed a large ulcerated area on the top of her head. Initially, this lesion was believed to be consistent with another pyoderma gangrenosum lesion. She was treated with steroids and dapsone, but the lesion showed no improvement. This area eventually measured about $8 \mathrm{~cm}$ across (Figure 1(a)). There were a number of subcutaneous or "satellite" metastases, some many centimeters distant. Therefore, she was referred to a dermatologist in the spring of 2012. Biopsies of lesions showed a spindle cell neoplasm (Figure 2(a)). Tumor cells strongly expressed CD10 (Figure 2(b)) and weakly expressed smooth muscle antigen. It did not stain for S100, cytokeratin, CD34, and desmin. It was also found that the neoplasm had invaded the adipose tissue and showed signs of necrosis.

During a clinic visit on June 6, 2012, the patient was invited to participate in an IRB approved protocol designed to evaluate a novel method for culturing circulating tumor cells from cancer patients. This study is reviewed and approved annually by the University Medical Center of Southern Nevada IRB and is registered in the National Cancer Institute (NCI) clinical trials database (http://www.clinicaltrials.gov/show/NCT01528774). Following signed informed consent, $10 \mathrm{ml}$ of anticoagulated blood was drawn into a standard vacutainer tube.

Anthracyclines have been the mainstay of systemic therapy for treatment metastatic soft tissue sarcoma since the 1970's. Other drugs that are thought to have modest clinical activity as single agents are ifosfamide, epirubicin, gemcitabine, and paclitaxel [6] [10] [11]. Our patient was treated with two cycles of epirubicin/ifosfamide chemotherapy [12]. The first cycle produced fever and neutropenia despite an initial conservative $75 \%$ dose. With supportive antibiotics and filgrastim, the patient recovered uneventfully. Following recovery of her blood counts, the patient was treated with a second cycle of chemotherapy with further dose reduction. Immediately after this treatment, the patient unexpectedly developed a bowel perforation requiring an exploratory laparotomy. Her post-operative recovery was further delayed by subsequent development of neutropenic fever, and severe pan-colitis and pan-enteritis. After many weeks in the hospital and subsequently in a rehabilitation facility, she improved enough to return home. Despite intensive chemotherapy, the scalp AFX lesion failed to respond. A second CTC-derived colony assay, performed on October 22, 2012, revealed that her tumor cell colony count spiked to $>376$ colonies (Figure 3 ).

Due to medical frailty and inability to tolerate further chemotherapy or radiotherapy, the patient was started on oral pazopanib on January 8, 2013 as palliative treatment. This treatment produced dramatic disease regression (Figure 1(b)). This area of regressed tumor was re-biopsied (Figure 2(c), Figure 2(d)). After a number of months, the dosage of pazopanib was reduced to $600 \mathrm{mg}$, and then to $400 \mathrm{mg} /$ day due to diarrhea and fatigue. A third CTC-derived colony assay was performed after 11 months on pazopanib therapy. This repeat CTC culture assay grew 8 colonies $/ 10 \mathrm{ml}$ blood (Figure 3 ), showing a strong correlation with both the clinical course and biopsy results. Due to the dramatic clinical response, the patient has been maintained on low dose pazopanib therapy for over 43 months with ongoing complete response. 


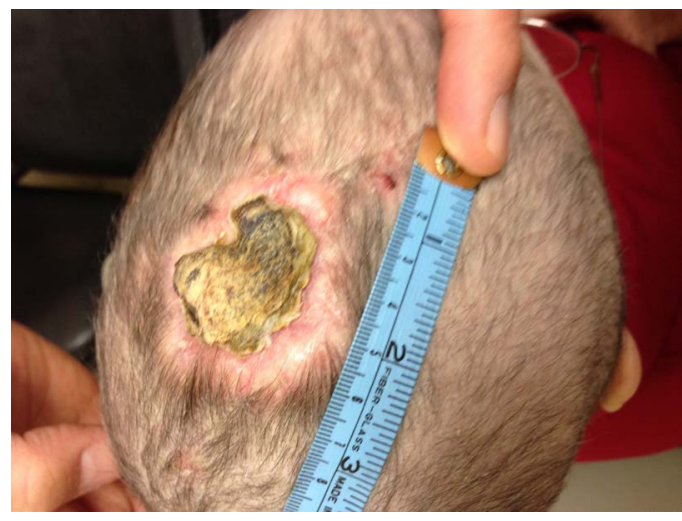

(a)

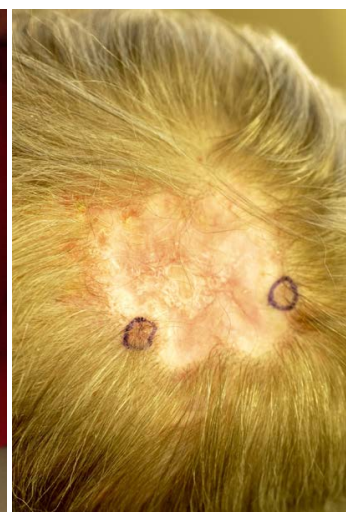

(b)

Figure 1. Clinical appearance of the patient's tumor. (a) Pretreatment photograph. (b) Post-treatment photograph. Rebiopsy sites marked.

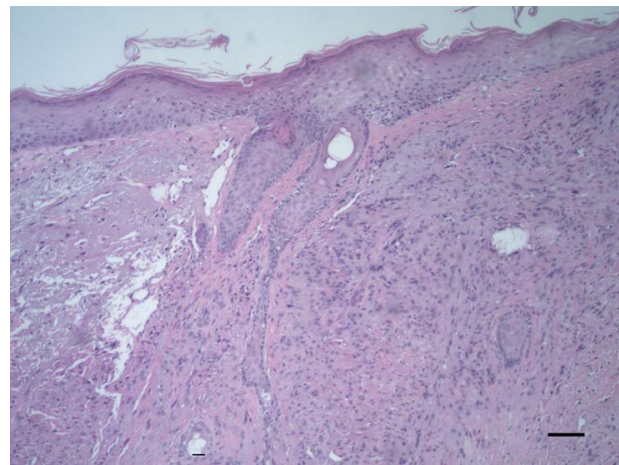

(a)

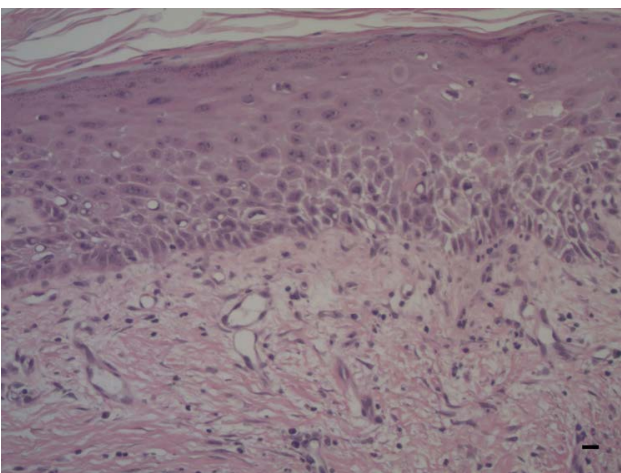

(c)

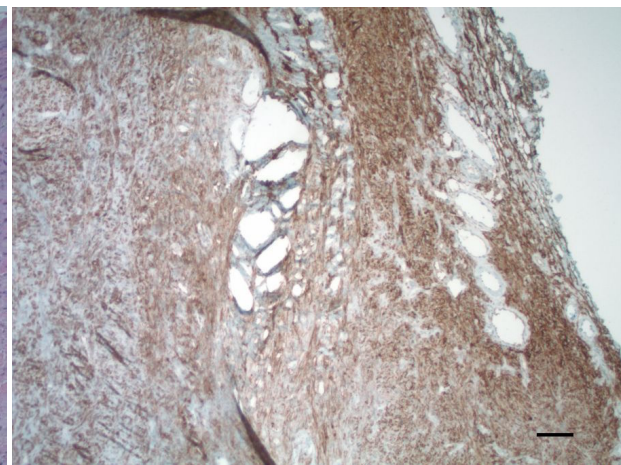

(b)

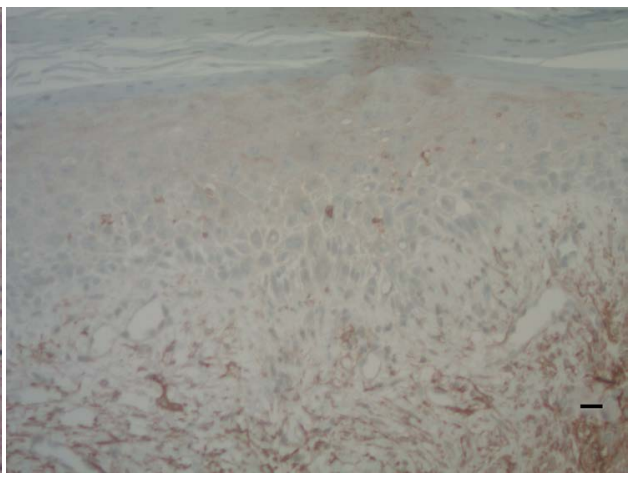

(d)

Figure 2. Photomicrographs of patient biopsies. (a) Pretreatment biopsy hematoxylin and eosin stain, subdermal invasion of anaplastic tumor cells tumor $(10 \times$, bar $50 \mu \mathrm{m})$. (b) CD10 stain of tumor, showing typical staining pattern $(4 \times$, bar $50 \mu \mathrm{m})$. (c) Post-treatment rebiopsy demonstrating scar without residual viable tumor (H \& E stain, 100×, bar $10 \mu \mathrm{m}$ ). (d) CD10 staining of post-treatment biopsy $(100 \times$, bar $10 \mu \mathrm{m})$.

\section{Discussion}

Due to the size of the primary tumor in this patient, invasion into adipose tissue, tumor necrosis, and presence of in-transit metastases we believe our patient fits the current 


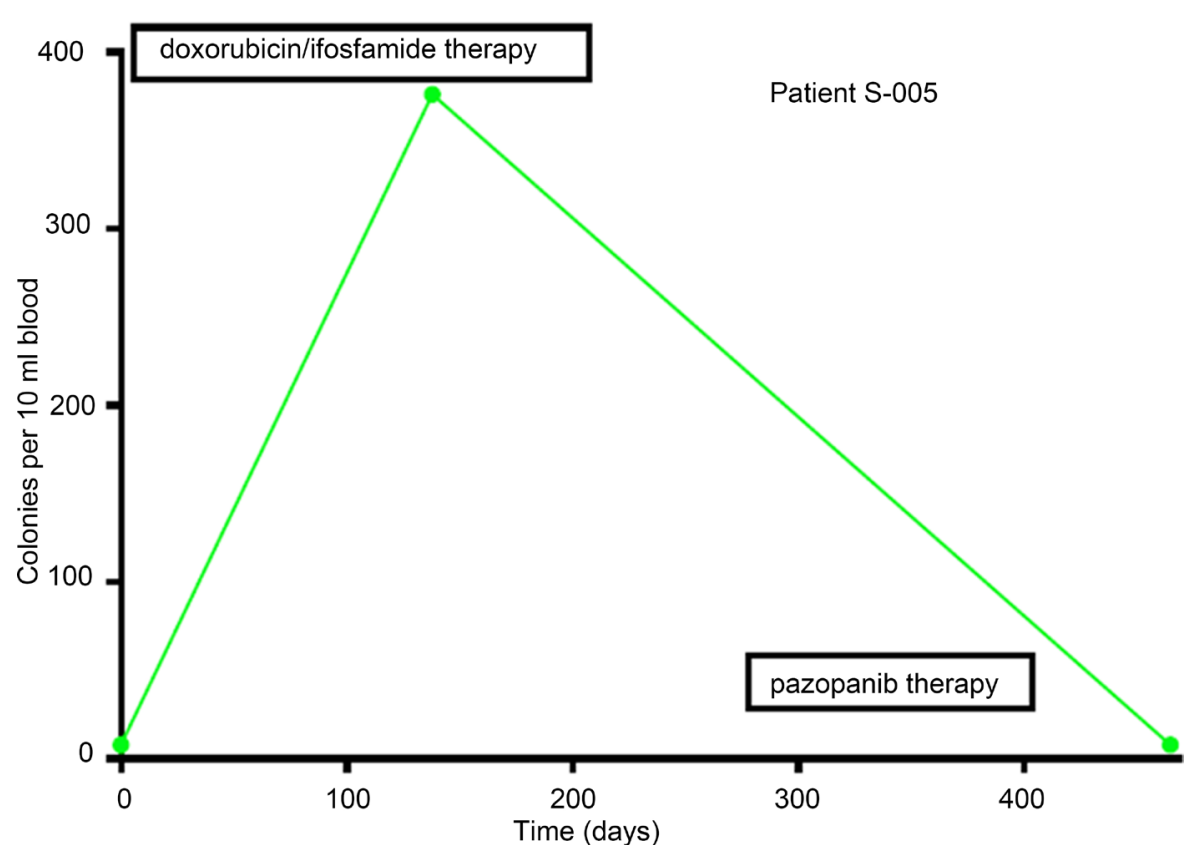

Figure 3. Circulating tumor cell-derived colonies following chemotherapy and pazopanib therapy.

definition of PDS. The tumor also arose on a UV-exposed site, suggesting that it had potentially arisen from UV damage. There was no evidence of extradermal primary or metastatic tumor, excluding UPS. The natural history of these tumors is aggressive local growth, with potential for distant metastases (as in this patient) contributing to the eventual demise. These cancers are generally treated with wide surgical excision, whenever possible. Little is known about treatment of metastatic PDS. Generally standard sarcoma chemotherapy regimens, such as adriamycin \pm ifosfamide or gemcitibine + taxane have been used, resulting in low response rates. Long term responses or remissions are generally not achieved with these approaches.

Angiogenesis is of crucial importance to the pathogenesis of many cancers making, research into anti-angiogenic agents has proven especially attractive. Pazopanib is a tyrosine kinase inhibitor that targets multiple angiogenic receptors. It predominantly targets vascular endothelial growth factor receptors (VEGFRs). However, there are also inhibitory effects on c-KIT, fibroblast growth factor (FGFR), and platelet derived growth factor (PDGFR).

Pazopanib has demonstrated significant clinical activity in non-adiopocyte derived soft-tissue sarcomas. In a recent randomized clinical trial, metastatic or unresectable sarcoma patients were randomly assigned to receive pazopanib $(\mathrm{n}=246)$ or placebo $(\mathrm{n}=$ 123) after failure of anthracycline based therapy [13]. Median progression-free survival was improved for pazopanib compared to placebo, although overall survival was not affected. Furthermore, it is notably more tolerable than traditional anthracycline-based chemotherapy. Potential toxicities associated with pazopanib include dose-related fatigue, diarrhea, nausea/vomiting, weight loss, and hypertension representing the most frequent 
side effects. Laboratory toxicities have included mild myelosuppression, hypophosphatemia and liver toxicity.

The PDS tumors in our patient did not respond at all to traditional anthracycline based chemotherapy, which resulted in life-threatening toxicty. However, a novel observation is that the PDS in this patient responded dramatically and with minimal side effects to an anti-angiogenic agent, pazopanib. This resulted in a biopsy-proven complete remission, so far lasting over 43 months with ongoing pazopanib therapy.

At some point during tumor development, malignant cells gain the ability to enter the vasculature, circulate, extravasate and grow in distant organs [14]. These circulating tumor cells (CTC) are believed to form metastases in vital organs, which remain the major cause of cancer-related mortality. CTC are cells that have arisen from either primary or metastatic tumor sites and have been released into the bloodstream. We believe that detection of tiny numbers of CTCs in the peripheral blood of cancer patients provides prognostic and predictive information related to patient survival and response to therapy survival due to their capacity to seed distant organs.

The traditional CTC counting systems pioneered by Veridex utilized the presence of epithelial cell adhesion molecules (EpCAM) expressed on the cell surface membrane of CTCs [15]. These markers are not expressed on normal blood cells. Using anti-EpCAM coated magnetic beads, CTCs can be extracted from the blood, counterstained with other epithelial markers, and counted via semi-automated fluorescence microscope scanning on glass slides [16] [17]. This approach has limited the evaluation of CTC to cancers that express EpCAM, such as breast, lung, prostate, and colon cancer. Other cancers, such as sarcoma, melanoma and renal cancer do not express this marker and are currently undetectable using the original Veridex assay [18].

We have developed a rapid, efficient, and inexpensive strategy for culturing CTC-derived colonies from a high percentage ( 90\%) of metastatic cancer patients [9]. This technology allows viable CTC-derived tumor cells to be isolated and expanded, providing suitable numbers of cancer cells for subsequent genetic or molecular analysis within 18 days of blood sampling. It should be noted that we have been able to culture CTC-derived colonies from a broad variety of cancers, including breast, lung, prostate, melanoma, sarcoma, renal cancer and others.

\section{Conclusion}

In conclusion, very little information is available about optimal treatment of metastatic AFX lesions. We demonstrated that pazopanib is a potentially active agent in locally advanced or metastatic AFX tumors. In addition, CTC-derived cultures of the unfractionated buffy coat of peripheral blood should be further investigated as a potential prognostic marker in sarcoma.

\section{References}

[1] Sakamoto, A. (2008) Atypical Fibroxanthoma. Clinical Medicine Oncology, 2, 117-127.

[2] Gleason, B.C. and Fletcher, C.D. (2008) Deep “Benign” Fibrous Histiocytoma: Clinicopa- 
thologic Analysis of 69 Cases of a Rare Tumor Indicating Occasional Metastatic Potential. The American Journal of Surgical Pathology, 32, 354-362.

http://dx.doi.org/10.1097/PAS.0b013e31813c6b85

[3] Mirza, B. and Weedon, D. (2005) Atypical Fibroxanthoma: A Clinicopathological Study of 89 Cases. Australasian Journal of Dermatology, 46, 235-238.

http://dx.doi.org/10.1111/j.1440-0960.2005.00190.x

[4] Dettrick, A. and Strutton, G. (2006) Atypical Fibroxanthoma with Perineural or Intraneural Invasion: Report of Two Cases. Journal of Cutaneous Pathology, 33, 318-322. http://dx.doi.org/10.1111/j.0303-6987.2006.00412.x

[5] Miller, K., Goodlad, J.R. and Brenn, T. (2012) Pleomorphic Dermal Sarcoma: Adverse Histologic Features Predict Aggressive Behavior and Allow Distinction from Atypical Fibroxanthoma. The American Journal of Surgical Pathology, 36, 1317-1326.

http://dx.doi.org/10.1097/PAS.0b013e31825359e1

[6] Maki, R.G., Wathen, J.K., Patel, S.R., Priebat, D.A., Okuno, S.H., Samuels, B., et al. (2007) Randomized Phase II Study of Gemcitabine and Docetaxel Compared with Gemcitabine alone in Patients with Metastatic Soft Tissue Sarcomas: Results of Sarcoma Alliance for Research through Collaboration Study 002. Journal of Clinical Oncology, 25, 2755-2763. http://dx.doi.org/10.1200/JCO.2006.10.4117

[7] Satter, E.K. (2012) Metastatic Atypical Fibroxanthoma. Dermatology Online Journal, 18, 3.

[8] Matushansky, I., Charytonowicz, E., Mills, J., Siddiqi, S., Hricik, T. and Cordon-Cardo, C. (2009) MFH Classification: Differentiating Undifferentiated Pleomorphic Sarcoma in the 21st Century. Expert Review of Anticancer Therapy, 9, 1135-1144. http://dx.doi.org/10.1586/era.09.76

[9] Samlowski, W.E., McGregor, J.R., Samlowski, S.T., Tharkar, S., Shen, S. and Bentz, J.S. (2014) Growth of Circulating Tumor Cell-Derived Colonies from Peripheral Blood of Melanoma Patients: Preliminary Characterization of Colony Composition. Health Psychology, 6, 1467-1481.

[10] Lorigan, P., Verweij, J., Papai, Z., Rodenhuis, S., Le Cesne, A., Leahy, M.G., et al. (2007) Phase III Trial of Two Investigational Schedules of Ifosfamide Compared with StandardDose Doxorubicin in Advanced or Metastatic Soft Tissue Sarcoma: A European Organisation for Research and Treatment of Cancer Soft Tissue and Bone Sarcoma Group Study. Journal of Clinical Oncology, 25, 3144-3150.

http://dx.doi.org/10.1200/JCO.2006.09.7717

[11] Nielsen, O.S., Dombernowsky, P., Mouridsen, H., Crowther, D., Verweij, J., Buesa, J., et al. (1998) High-Dose Epirubicin Is Not an Alternative to Standard-Dose Doxorubicin in the Treatment of Advanced Soft Tissue Sarcomas. A Study of the EORTC Soft Tissue and Bone Sarcoma Group. British Journal of Cancer, 78, 1634-1639. http://dx.doi.org/10.1038/bjc.1998.735

[12] Ryan, C.W., Montag, A.G., Hosenpud, J.R., Samuels, B., Hayden, J.B., Hung, A.Y., et al. (2008) Histologic Response of Dose-Intense Chemotherapy with Preoperative Hypofractionated Radiotherapy for Patients with High-Risk Soft Tissue Sarcomas. Cancer, 112, 2432-2439. http://dx.doi.org/10.1002/cncr.23478

[13] van der Graaf, W.T., Blay, J.Y., Chawla, S.P., Kim, D.W., Bui-Nguyen, B., Casali, P.G., et al. (2012) Pazopanib for Metastatic Soft-Tissue Sarcoma (PALETTE): A Randomised, DoubleBlind, Placebo-Controlled Phase 3 Trial. The Lancet, 379, 1879-1886. http://dx.doi.org/10.1016/S0140-6736(12)60651-5

[14] Bernards, R. and Weinberg, R.A. (2002) A Progression Puzzle. Nature, 418, 823.

[15] Alunni-Fabbroni, M. and Sandri, M.T. (2010) Circulating Tumour Cells in Clinical Prac- 
tice: Methods of Detection and Possible Characterization. Methods, 50, 289-297.

http://dx.doi.org/10.1016/j.ymeth.2010.01.027

[16] Allard, W.J., Matera, J., Miller, M.C., Repollet, M., Connelly, M.C., Rao, C., et al. (2004) Tumor Cells Circulate in the Peripheral Blood of All Major Carcinomas but Not in Healthy Subjects or Patients with Nonmalignant Diseases. Clinical Cancer Research, 10, 6897-6904. http://dx.doi.org/10.1158/1078-0432.CCR-04-0378

[17] Cristofanilli, M., Hayes, D.F., Budd, G.T., Ellis, M.J., Stopeck, A., Reuben, J.M., et al. (2005) Circulating Tumor Cells: A Novel Prognostic Factor for Newly Diagnosed Metastatic Breast Cancer. Journal of Clinical Oncology, 23, 1420-1430.

http://dx.doi.org/10.1200/JCO.2005.08.140

[18] Balzar, M., Winter, M.J., de Boer, C.J. and Litvinov, S.V. (1999) The Biology of the 17-1A Antigen (Ep-CAM). Journal of Molecular Medicine, 77, 699-712.

http://dx.doi.org/10.1007/s001099900038

\section{Submit or recommend next manuscript to SCIRP and we will provide best service} for you:

Accepting pre-submission inquiries through Email, Facebook, LinkedIn, Twitter, etc. A wide selection of journals (inclusive of 9 subjects, more than 200 journals)

Providing 24-hour high-quality service

User-friendly online submission system

Fair and swift peer-review system

Efficient typesetting and proofreading procedure

Display of the result of downloads and visits, as well as the number of cited articles

Maximum dissemination of your research work

Submit your manuscript at: http://papersubmission.scirp.org/

Or contact jet@scirp.org 